\title{
Research Article Electrical Properties of Self-Assembled Nano-Schottky Diodes
}

\author{
F. Ruffino, ${ }^{1,2}$ A. Canino, ${ }^{1,2}$ M. G. Grimaldi, ${ }^{1,2}$ F. Giannazzo, ${ }^{3}$ F. Roccaforte, ${ }^{3}$ and V. Raineri ${ }^{3}$ \\ ${ }^{1}$ Dipartimento di Fisica ed Astronomia, Università di Catania, via Santa Sofia 64, 95123 Catania, Italy \\ ${ }^{2}$ MATIS CNR-INFM, Department of Physics and Astronomy, University of Catania, via Santa Sofia 64, 95123 Catania, Italy \\ ${ }^{3}$ Consiglio Nazionale delle Ricerche-Istituto per la Microelettronica e Microsistemi (CNR-IMM), Stradale Primosole 50, \\ 95121 Catania, Italy
}

Correspondence should be addressed to F. Ruffino, francesco.ruffino@ct.infn.it

Received 23 May 2008; Accepted 9 September 2008

Recommended by Xuedong Bai

A bottom-up methodology to fabricate a nanostructured material by Au nanoclusters on $6 \mathrm{H}$-SiC surface is illustrated. Furthermore, a methodology to control its structural properties by thermal-induced self-organization of the Au nanoclusters is demonstrated. To this aim, the self-organization kinetic mechanisms of Au nanoclusters on SiC surface were experimentally studied by scanning electron microscopy, atomic force microscopy, Rutherford backscattering spectrometry and theoretically modelled by a ripening process. The fabricated nanostructured materials were used to probe, by local conductive atomic force microscopy analyses, the electrical properties of nano-Schottky contact Au nanocluster/SiC. Strong efforts were dedicated to correlate the structural and electrical characteristics: the main observation was the Schottky barrier height dependence of the nano-Schottky contact on the cluster size. Such behavior was interpreted considering the physics of few electron quantum dots merged with the concepts of ballistic transport and thermoionic emission finding a satisfying agreement between the theoretical prediction and the experimental data. The fabricated Au nanocluster/SiC nanocontact is suggested as a prototype of nano-Schottky diode integrable in complex nanoelectronic circuits.

Copyright ( 92008 F. Ruffino et al. This is an open access article distributed under the Creative Commons Attribution License, which permits unrestricted use, distribution, and reproduction in any medium, provided the original work is properly cited.

\section{INTRODUCTION}

Understanding the effects of downscaling the devices dimensions to the nanometer size is one of the most important topics in the modern material science applied to microelectronics. In fact, the confinement of electrons in dimensions typical of atoms and molecules obliges to consider their quantum behavior. Therefore, a new class of effects are characterizing ultrascaled devices. In the last years, these ideas led to the birth of the "nanotechnology and nanoelectronic revolution" [1-4] with the aim to understand the effects of downscaling the matter in the atomic range and to develop innovative nanostructured materials and quantum effects based devices [1-4] following a bottom-up procedure with respect to the traditional top-down scaling scheme.

In particular, the nanometric level knowledge of the structural characteristics of such innovative materials and the nanometric control and manipulation of these characteristics acquired a fundamental importance in the design and realization of innovative electrical nanodevices. In fact, it is well known that the local electrical characteristics of such devices are dramatically dependent on the local structural ones. Hence, a precise control and manipulation (at atomic level) of the structural characteristics allow the precise control and manipulation of the electrical ones that are always innovative properties with respect to the traditional devices.

A promising topic of nanotechnology research is, surely, the study of the structural and electrical properties of nanometric metal clusters deposited on or embedded in semiconductor/insulating substrates in view of the realization of nanostructured materials with electrical properties dependent on and tuned by the structural ones (clusters size, density, etc.) [5].

We developed a methodology to control and manipulate the clusters structural properties based on the selforganization mechanism of the Au nanoclusters (NCs) on the $\mathrm{SiC}$ surface induced by thermal processes. The Au clustering is shown to be a ripening process of three-dimensional structures controlled by surface diffusion and the application 
of the ripening theory enabled us to derive the surface diffusion coefficient and all other parameters necessary to describe the entire process so that we achieved a control on size, size distribution, clusters distance distribution, and surface fraction of area covered by the clusters by simply controlling the process parameters.

We suggest to apply the self-organization of Au NCs as a nanotechnology step to fabricate innovative nanostructured devices. For main example, we studied, by the conductive atomic force microscopy (C-AFM) technique, the local electrical properties of the nanometric systems $\mathrm{Au} \mathrm{NC} / \mathrm{SiC}$ substrate. As expected, the main result was the strongly dependence of the electrical properties on the clusters size, density, fraction of covered area. In particular, we observed the Schottky barrier height dependence of the $\mathrm{Au} \mathrm{NC/SiC}$ nanocontact on the cluster size. Furthermore, we propose a model to interpret such a behavior.

\section{EXPERIMENT}

$6 \mathrm{H}-\mathrm{SiC}$ substrates (previously etched in $10 \%$ aqueous $\mathrm{HF}$ solution to remove the native oxide) were used. A set of substrates was covered by a $2 \mathrm{~nm}$ (nominally) thick Au layer sputtered using an Emitech K550x Sputter coater apparatus (Ar plasma, $10^{-6}$ mbar). The samples so obtained were named "as-deposited samples." Some as-deposited samples were then annealed in Ar at different temperatures $(873 \mathrm{~K} \div$ $1073 \mathrm{~K}$ ) for several times (5 minutes $\div 60$ minutes) and analyzed by Rutherford backscattering spectrometry (RBS), atomic force microscopy (AFM), and scanning electron microscopy (SEM).

The RBS analyses were performed using $2 \mathrm{MeV}{ }^{4} \mathrm{He}^{+}$ backscattered ions at $165^{\circ}$. The AFM analyses were performed using a digital instruments microscope dimension 3100 in high amplitude mode and ultrasharpened Si tips were used and substituted as soon as a resolution loose was observed during the acquisition. The AFM images were analyzed by using the Nanoscope III software. The SEM analyses were performed by a Zeiss FEG-SEM Supra 25 Microscope and the SEM images were analyzed using the Gatan Digital Micrograph software.

The local transversal current-voltage $\left(I-V_{\text {tip }}\right)$ analyses were carried out at room temperature using the Veeco DI 3100 AFM, in contact mode, equipped with the conductiveAFM (C-AFM) head and ultrasharpened diamond coated Si tips. The conductive diamond coating is polycrystalline and the effective tip diameter is ultimately set by the very small diamond grain (a few nanometers) placed at the apex of the tip. For each sample, $400 I-V$ acquisitions in 400 different positions were performed in a matrix of $20 \times 20$ points with step of $500 \mathrm{~nm}$.

\section{RESULTS AND DISCUSSIONS}

\subsection{Self-organization of Au nanoclusters on hexagonal SiC surface}

RBS analyses allowed to determine, in particular, the Au atomic concentration $Q$ in the samples and it gave the same result (within a statistical error of 5\%) for all the samples (as-deposited and annealed) and its value is $Q=4.5 \times$ $10^{15} \mathrm{Au} / \mathrm{cm}^{2}$. So we can conclude that no $\mathrm{Au}$ loss occurs during thermal treatments for any of our samples (out diffusion, evaporation, reaction with $\mathrm{C}$ and/or $\mathrm{Si}$ ).

The change in morphology has been followed by AFM. Despite a tip-cluster, deconvolution was considered; surface morphology can present some artifacts derived by the tipNCs interaction (not allowing an accurate determination of NCs shape and dimension). So, for a supplementary accuracy, we compared the information acquired by AFM with the NCs images obtained by SEM. From the AFM and SEM images, the NCs size distributions and the distributions of center-to-center distances between nearest NCs were determined by using a software that defines each NC area by the surface image sectioning of a plane that was positioned at half NC height. However, the results obtained by AFM and SEM analyses are in good agreement (the respective results are identical within the statistical error). So, AFM and SEM analyses were crossed to derive NC size distributions and center-to-center NC distance distributions.

As an example, Figures 1(a), 1(b) show representative AFM and SEM images for the as-deposited sample and Figures 1(c), 1(d) for the $1073 \mathrm{~K}-60$ minutes sample.

In particular, the mean $\mathrm{NC}$ radius $\langle R(t)\rangle$ was derived for each examined annealing temperature and time by the obtained NC size distribution. Furthermore, if $V(T, t)=$ $(4 / 3) \pi\langle R\rangle^{3}$ is the mean volume of the NC for each annealing temperature $T$ and annealing time $t$, being [6] $\Omega=$ $1.69 \times 10^{-29} \mathrm{~m}^{3}$ the Au atomic volume, then $\langle n\rangle=\langle V\rangle / \Omega$ (supposing a $100 \%$ packing density) is the mean number of Au atoms forming the NC. Therefore, $N_{s}(T, t)=Q /\langle n\rangle$ is the mean number of NC per unit area and $F(T, t)=$ $\pi R^{2}(T, t) N_{s}(T, t)$ the covered area by the NCs. The obtained experimental $F(T, t)$ is showed (dots) in Figure 2(a).

In the following, we briefly recall the kinetic growth evolution of the NCs by the coarsening (or ripening) model to explain the observed self-organization mechanism of the $\mathrm{Au}$ NCs on the SiC surface.

At any stage during coarsening there is a so-called critical particle radius $R^{*}$ being in equilibrium with the mean matrix composition; particles with $R>R^{*}$ will grow and particles with $R<R^{*}$ will shrink [7]. Many coarsening theories are presented in literature [7-10]. In particular, the concepts that we report here (which are directly connected with our experimental situations and data) are primarily based on the review works of Baldan [7] and the theoretical work of Lifshitz-Slyozov-Wagner [8, 9] (LSW theory), AllmangFeldman-Grabov [10].

The aim of mathematical modeling of ripening process of particles dispersed ( $\mathrm{Au}$ in our case) in/on a matrix is to calculate the growth rate of individual particle. Despite the particular differences derived by the boundary conditions relative to the particular case examined, the general theory of ripening process based on the LSW ideas has the same result for the asymptotic temporal evolution of the system, and is summarized as follows [10]: the mean particle radius $R$ evolves as a function of time (for time sufficiently great, 


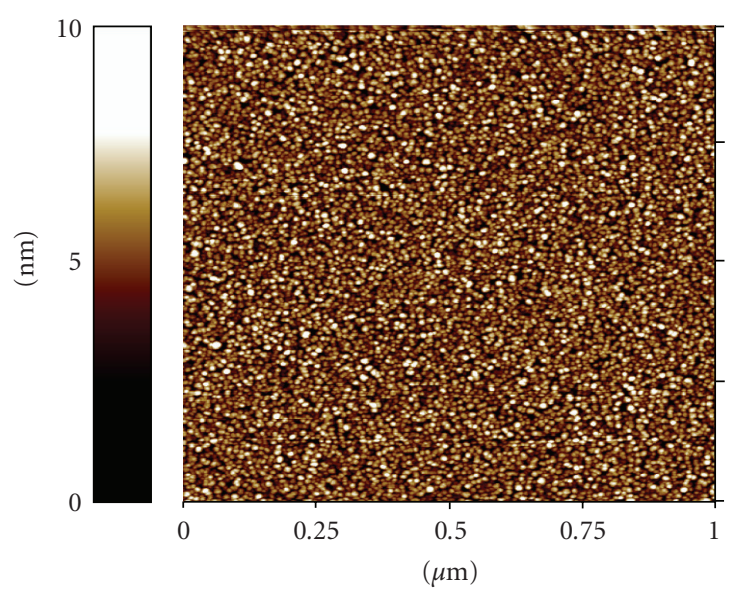

(a)

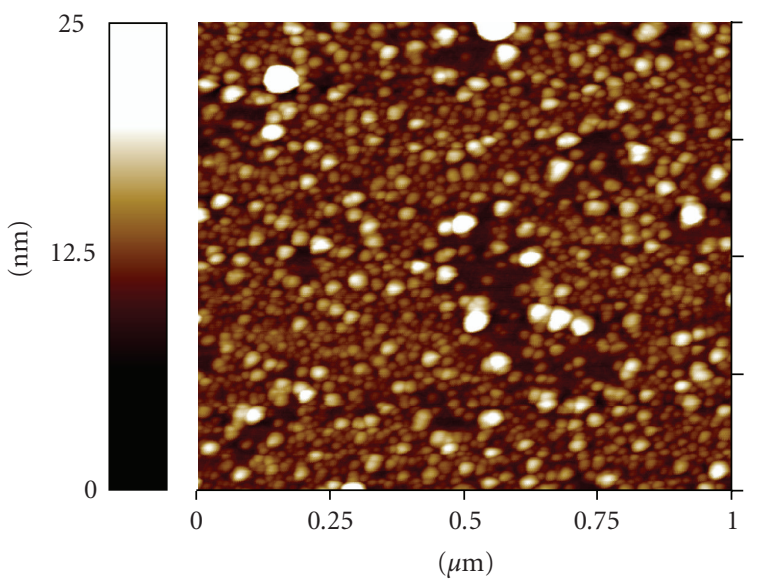

(c)

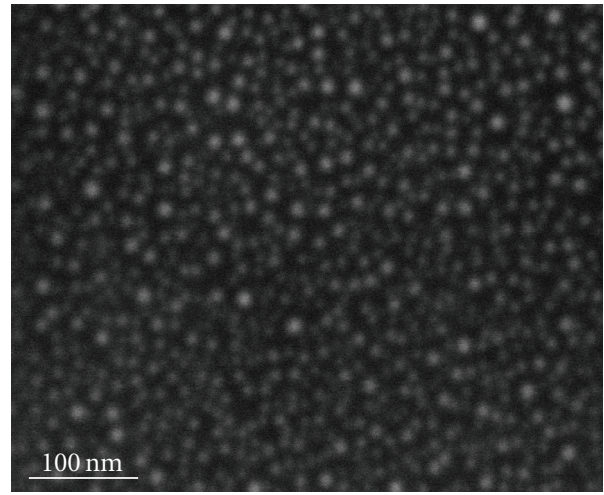

(b)

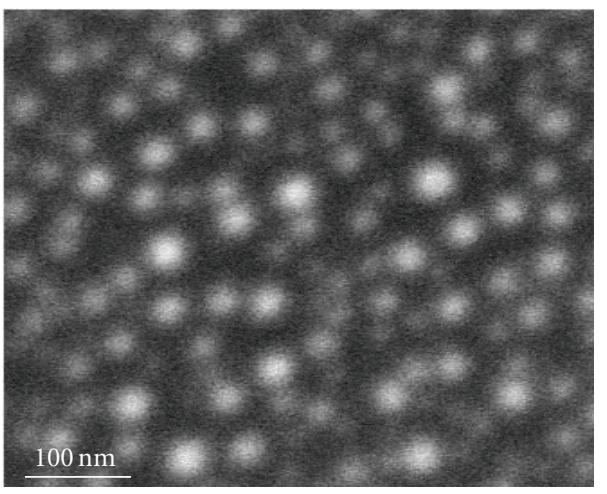

(d)

FIGURE 1: (a) AFM image of the Au clusters as-deposited on 6H-SiC substrate, (b) SEM image for the same sample, (c) AFM image of the Au clusters as-deposited on 6H-SiC substrate and annealed $1073 \mathrm{~K}-60$ minutes, (d) SEM image for the same sample.

i.e., in stationary state) according to

$$
R^{n}(t)-R_{0}^{n}=K^{*} t
$$

with $R_{0}$ the radius of the particle at time $t=0$, and $K^{*}$ an appropriate constant depending on the diffusion coefficient. In particular, the fundamental formulations of the ripening theory differ themselves by the value of $n: n=2(2 \mathrm{D} / 2 \mathrm{D}$ case) for the growth of two-dimensional (2D) particles on a surface (2D); $n=3$ (3D/3D case) for the growth of threedimensional (3D) particles embedded in a bulk matrix (3D); $n=4(3 \mathrm{D} / 2 \mathrm{D}$ case $)$ for the growth of three-dimensional (3D) particles on a surface (2D).

The Au on SiC has a strong nonwetting nature [11], being its adhesion energy on $\mathrm{SiC} E_{\text {adh }}(\mathrm{Au} / \mathrm{SiC})=445 \mathrm{~mJ} / \mathrm{m}^{2}$ [11] much lower than the Au surface energy $\gamma_{\mathrm{Au}}=1500 \mathrm{~mJ} / \mathrm{m}^{2}$ [12], so that the $\mathrm{Au} \mathrm{NCs}$ have grown on $\mathrm{SiC}$ as $3 \mathrm{D}$ structures.

According to this considerations, if the Au clustering on $\mathrm{SiC}$ surface, in the examined temperature range, is guided by a ripening process of $3 \mathrm{D}$ structures limited by diffusion, the temporal variation of the mean NC radius $\langle R\rangle$ should be regulated by (1) with $n=4$; it is demonstrated by the data reported in Figure 2(a). In such a figure are reported the experimental data $\langle R\rangle^{4}-\left\langle R_{0}\right\rangle^{4}$ (dots) for all the investigated temperature and the lines (continuous, dashed, and dotted) represent the theoretical fits by (1) (using $n=4$ ), being $K^{*}$ the fit parameter. The good agreement between the experimental data and the fits is evident.

Therefore, in the assessed growth modes, the mean radius of the Au NCs on $\mathrm{SiC}$ in the examined temperature and time ranges increases with time as indicated by (1) with $n=4$ and with $K^{*}$ defined by [13]

$$
K^{*}=\frac{8 N_{0} D_{s} \gamma \Omega^{2}}{45 k_{B} T \ln (L)}
$$

being $D_{s}$ the surface diffusion coefficient of the Au atoms on the $\mathrm{SiC}$ surface, $L \approx 3$ a characteristic length (in units of $\langle R\rangle$ ), $N_{0}$ the density of nucleation sites, $\Omega$ the Au atomic volume, $\gamma$ the Au surface energy $\left(N_{0}=1.22 \times 10^{15} \mathrm{~cm}^{-2}, \Omega=1.69 \times\right.$ $\left.10^{-29} \mathrm{~m}^{3}, \gamma=1.5 \mathrm{~J} / \mathrm{m}^{2}\right)[6,12]$. The fit of experimental data by (1) (with $n=4$ ), reported in Figure 2(a) for the different 


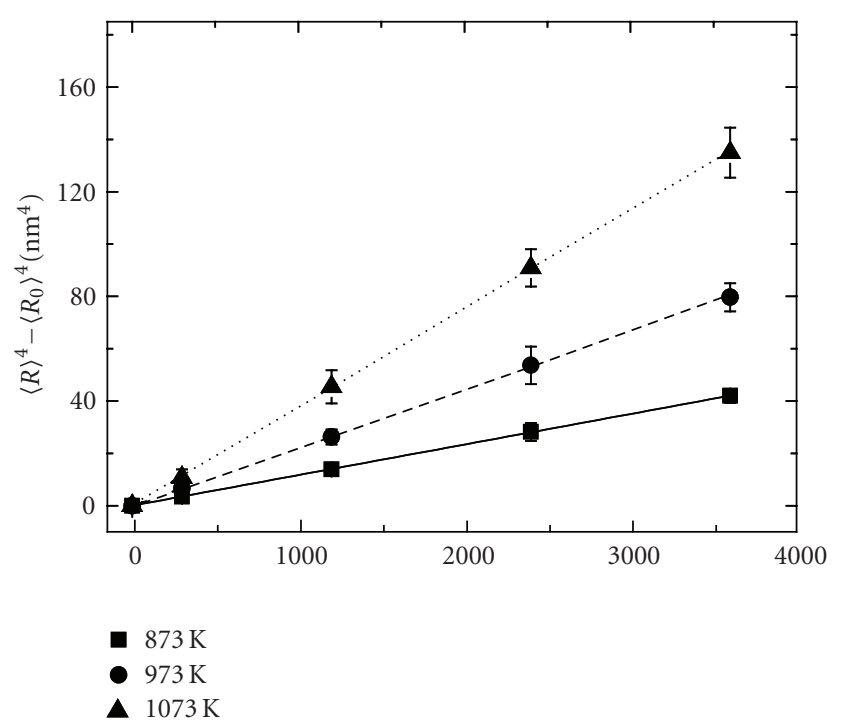

(a)

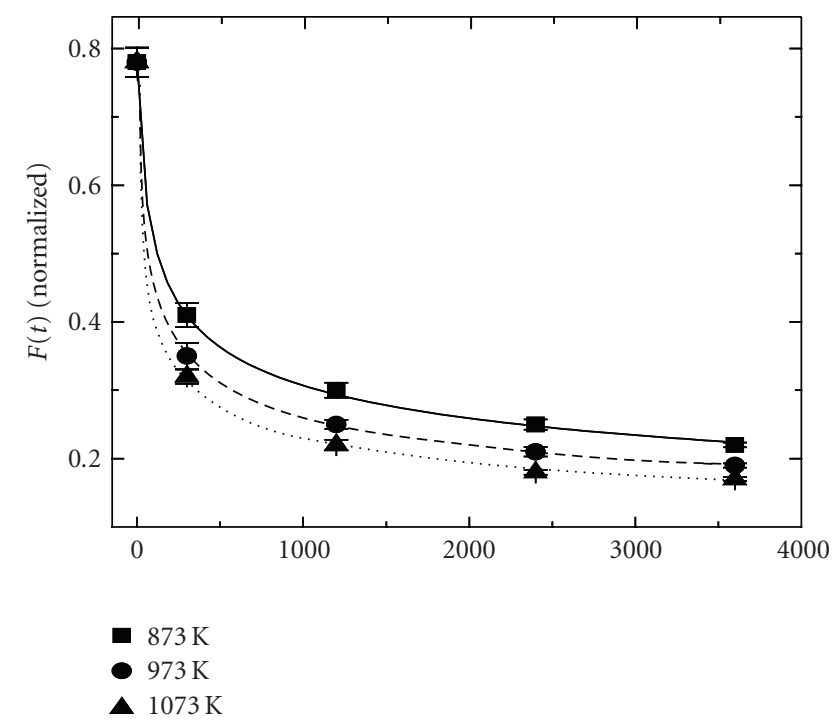

(b)

FIgURE 2: (a) Experimental $R^{4}-R_{0}^{4}$ (black dots) as a function of annealing time for each fixed temperature and the relative linear fits (continuous lines), being $K^{*}$ the fits parameter. (b) Experimental (dots) fraction $F$ (normalized) of surface area covered by clusters as a function of annealing time and relative theoretical simulation (curves).

annealing temperatures, allowed us to determine $K^{*}(T)$ for the three examined temperatures. Moreover, inversion of (2) allowed us to determine the diffusion coefficient of $\mathrm{Au}$ on SiC: $D_{s}(873 \mathrm{~K})=1.67 \times 10^{-15} \mathrm{~cm}^{2} / \mathrm{s}, D_{s}(973 \mathrm{~K})=3.52 \times$ $10^{-15} \mathrm{~cm}^{2} / \mathrm{s}, D_{s}(1073 \mathrm{~K})=6.58 \times 10^{-15} \mathrm{~cm}^{2} / \mathrm{s}$. Such values are consistent with an Arrhenius behavior $D_{s}(T)=D_{0} e^{-E_{a} / k_{B} T}$ so as predicted for a thermally activated diffusion process [14]. The fit of the experimental $D_{s}(T)$ with this activated form allows as to obtain the preexponential factor $D_{0}=(2.6 \times$ $\left.10^{-12} \pm 1.6 \times 10^{-13}\right) \mathrm{cm}^{2} / \mathrm{s}$ and the activation energy $E_{a}=$ $(0.55 \pm 0.01) \mathrm{eV} /$ atom.

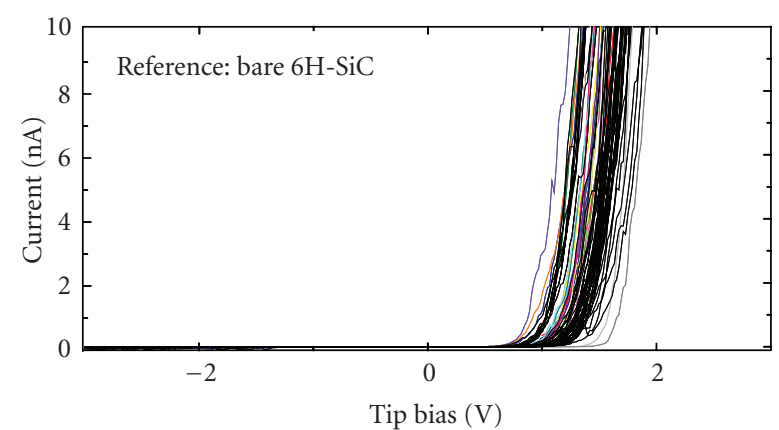

(a)

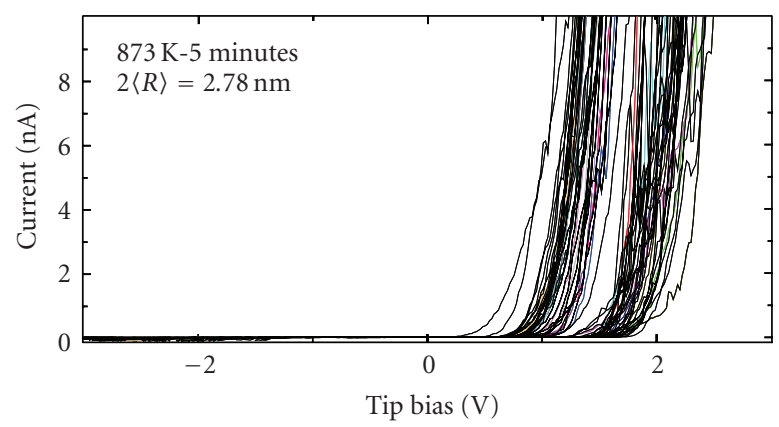

(b)

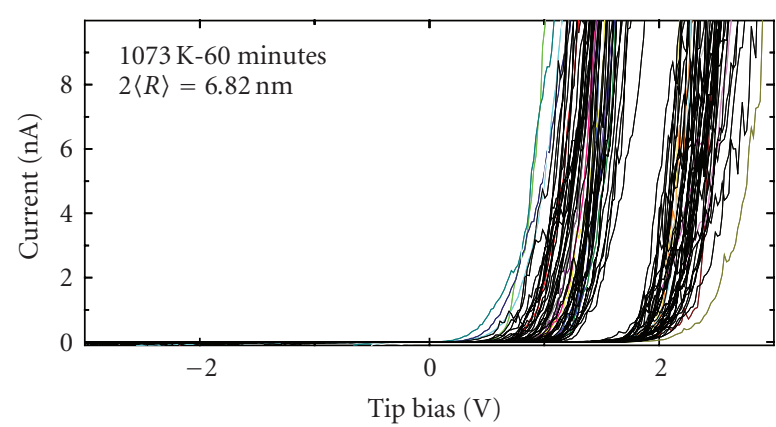

(c)

FIgURE 3: $I-V_{\text {tip }}$ curves measured (by C-AFM) in SiC covered with Au nanoclusters with different sizes.

Furthermore, the exposed model allows simulating the $F(t)$ behavior: in fact, in Figure 2(b) the continuous lines represent the prevision according to the exposed model and it is evident the good agreement with the experimental data.

\subsection{Electrical properties of the Au nanoclusters/SiC contacts}

The knowledge of the details of the self-organization mechanisms of $\mathrm{Au}$ NCs on SiC surfaces is a fundamental step towards innovative nanodevices design. For example, once the knowledge of the details of the Au NCs self-organization mechanisms on the $\mathrm{SiC}$ surface was established, we were able to probe the electrical behavior of the $\mathrm{Au} \mathrm{NCs} / \mathrm{SiC}$ 


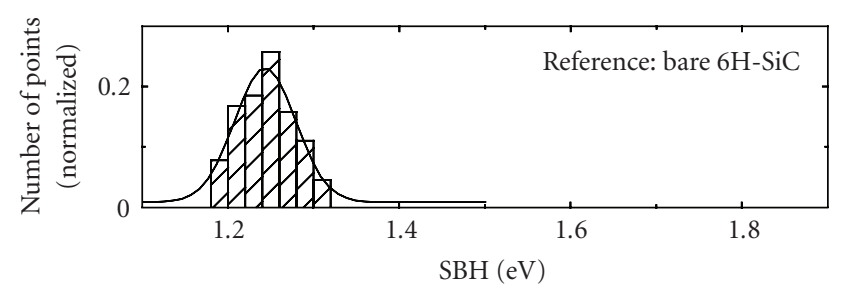

(a)

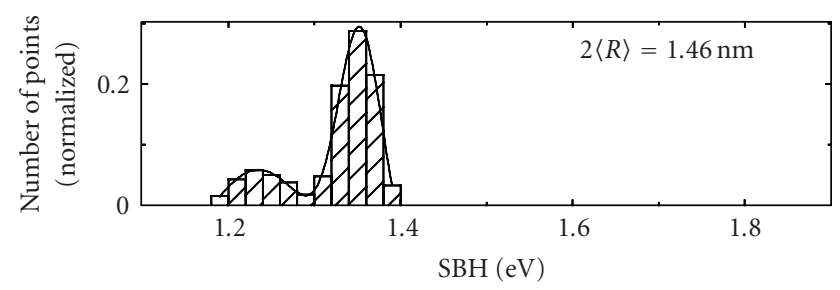

(b)

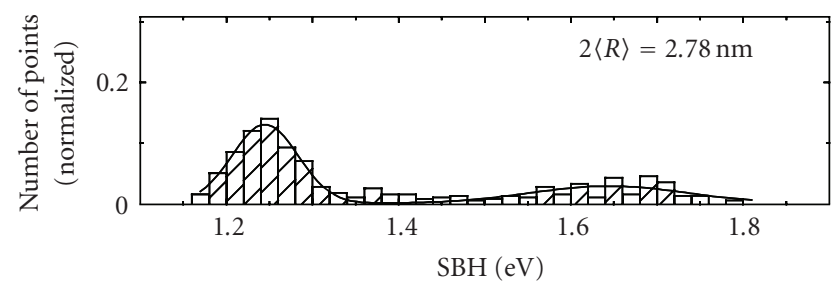

(c)

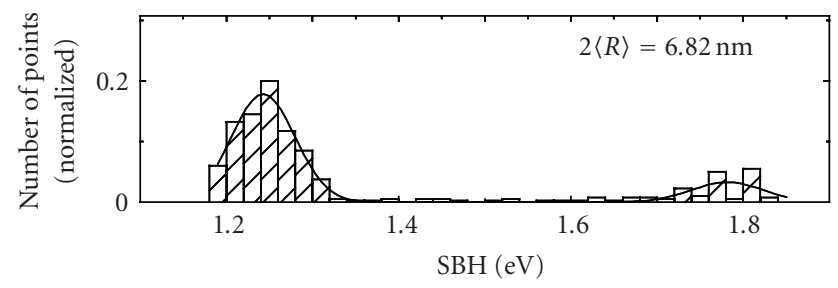

(d)

Figure 4: Schottky barrier height distributions. (a) Reference sample (sample without Au cluster); sample with Au cluster mean diameter of (b) $(1.46 \pm 0.03) \mathrm{nm},(\mathrm{c})(2.78 \pm 0.12) \mathrm{nm}$, and (d) $(6.82 \pm 0.12) \mathrm{nm}$.

nano-Schottky contacts observing their dependence on NCs size and fraction of area covered by cluster. Hence, by opportune annealing process we are able to control the structural properties of the fabricated nanostructured materials and, as a consequence, the electrical properties of nanodevices based on such systems.

The samples considered in these analyses were the asdeposited sample and the $873 \mathrm{~K}-5$ minutes, the $973 \mathrm{~K}-20$ minutes, the $973 \mathrm{~K}-60$ minutes, and the $1073 \mathrm{~K}-60$ minutes annealed samples. They showed NCs with mean diameter of $(1.46 \pm 0.03) \mathrm{nm},(2.78 \pm 0.12) \mathrm{nm},(4.54 \pm 0.11) \mathrm{nm}$, $(5.98 \pm 0.09) \mathrm{nm},(6.82 \pm 0.12) \mathrm{nm}$ and a mean center-tocenter distance between nearest NCs of $(4.31 \pm 0.18) \mathrm{nm}$, $(6.53 \pm 0.18) \mathrm{nm},(10.9 \pm 0.13) \mathrm{nm},(14.15 \pm 0.15) \mathrm{nm},(17.6 \pm$ $0.19) \mathrm{nm}$, respectively.

According to Giannazzo et al. [15], a biased C-AFM tip in contact with a continuous ultrathin metal film on a semiconductor forms a nano-Schottky diode due to the nanometric localization of the current across the metalsemiconductor (MS) interface. In our case of discontinuous film, for each tip position on the sample surface, the typical rectifying Schottky contact $I-V$ characteristics were found, with the threshold voltage (correlated to the Schottky barrier height (SBH)) depending on the tip position. As an example, in Figure 3 are compared the characteristics recorded in bare $\mathrm{SiC}$ (Figure 3(a)) and in $\mathrm{SiC}$ covered with $\mathrm{Au}$ NCs of different size (Figures 3(b), 3(c)). Each $I-V_{\text {tip }}$ curve is typical of thermoionic emission [16], and in the reference sample the $I-V_{\text {tip }}$ characteristics belong to a unique family that can be associated to the Schottky contact between the diamond tip and the $6 \mathrm{H}-\mathrm{SiC}$ substrate. In Au covered samples, the $I-V_{\text {tip }}$ curves are splitted in two families: one corresponds to the diamond/6H-SiC Schottky contact (area not covered by $\mathrm{Au}$ ) and the second one corresponds to the $\mathrm{Au} \mathrm{NC/SiC}$ Schottky contact. The second family shift towards higher voltage when the mean NCs size increases. To determine the $\mathrm{SBH}$, the current onset region of each $I-V$ curve was fitted with a parabolic function and the SBH value was determined as the parabola vertex derived by the fits, as described in [13]. So the SBH spatial distribution for each sample was obtained and reported in the normalized distributions of Figure 4(a) for the reference sample (sample without $\mathrm{Au}$ clusters). The SBH distribution is peaked at (1.24 \pm $0.02) \mathrm{eV}$, with a broadening due to statistical fluctuations. This measured value of $1.24 \mathrm{eV}$ is associated to the $\mathrm{SBH}$ of the diamond-tip/6H-SiC Schottky contact. For the samples with $\mathrm{Au}$ clusters on the surface, by increasing the mean cluster sizes, the $\mathrm{SBH}$ distributions exhibit a bimodal shape (see Figures 4(b), 4(c), 4(d)), with two broad peaks fitted by two Gaussian curves. For all the samples, the first peak is centred at $1.24 \mathrm{eV}$, that is, the value of the tip-diamond/6H$\mathrm{SiC} \mathrm{SBH}$. Hence, the presence of this first peak can be associated to the surface regions in which the diamond tip is directly in contact with the $6 \mathrm{H}-\mathrm{SiC}$ substrate. Interestingly, the position of the second peak changes with the NCs average dimension. The SBH values in the hystograms around the second peak can be associated to the direct contact with a single $\mathrm{Au} \mathrm{NC}$, that is, to a single Au NC/6H-SiC nanoSchottky diode. In fact, according to the used tip shape and to the average cluster-distance/cluster-dimension ratio (and the step of $500 \mathrm{~nm}$ between each point), it is quite unlikely that more than one cluster could be simultaneously contacted by the tip. Hence, when the tip is in contact with a single $\mathrm{NC}$, the nearest NCs can contribute to the total current only by a tunnel component through the air that is negligible, being, according to realistic estimations, at least two orders of magnitude smaller than the current due to the direct tip-NCsubstrate contact. Moreover, such a hypothesis is supported by the fact that the fraction of $I-V_{\text {tip }}$ curves belonging to the family in which the tip is in contact with Au corresponds to the fraction of covered area by Au NCs for that sample (Figure 5), measured by the structural analyses.

The data of Figure 4 demonstrate the dependence of the $\mathrm{SBH}$ on the NC size. As an example, in the as-deposited sample, the average cluster size is $\sim 1.46 \mathrm{~nm}$ with a Gaussian distribution (full width height maximum $\sigma=0.03 \mathrm{~nm}$ ). 


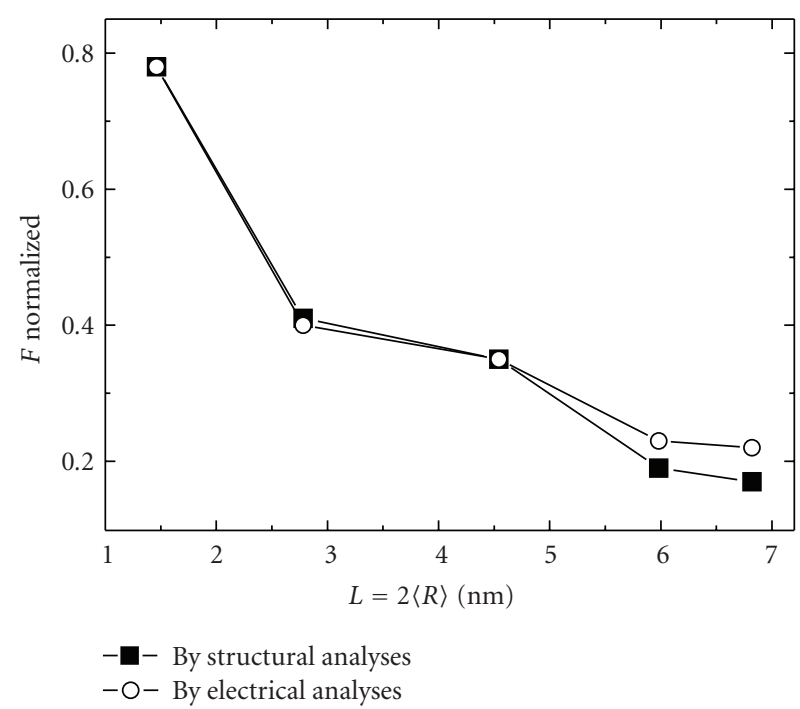

FIGURE 5: Comparison between the data concerning the fraction of covered area by the Au clusters (normalized) derived by the structural analyses (square dots) and by the electrical ones (circular dots).

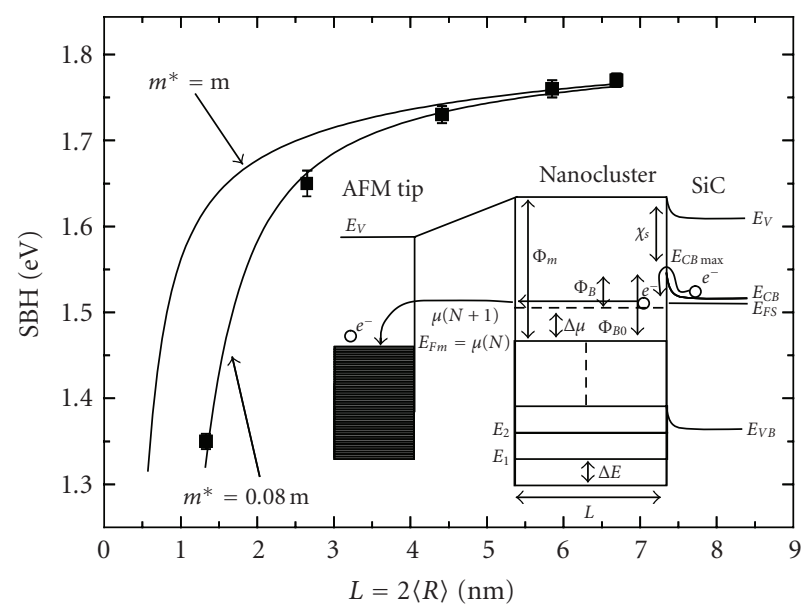

FIGURE 6: Experimental values (dots) of the $\mathrm{SBH}$ as a function of mean cluster size and theoretical prediction for $\Phi_{B}=1.85 \mathrm{eV}, m^{*}=$ 0.08 , and $m^{*}=m$. The inset shows the considered band diagram of the system (AFM) tip-cluster-SiC substrate.

Correspondingly, Figure 4(b) shows that most of NC/6H$\mathrm{SiC}$ contacts present a $\mathrm{SBH}$ around $1.35 \mathrm{eV}$ while only a low number of contacts present smaller or larger SBH. Similarly, in the other samples the peak corresponding to the tip- $\mathrm{Au}$ substrate contact is centred on the $\mathrm{SBH}$ value corresponding to the mean Au cluster size with dispersion due to the cluster dimensions. Accordingly, we associated to each sample a unique SBH corresponding to the cluster mean size (the peak at higher SBH in Figure 4) and the error bar on each SBH value was evaluated from the $\sigma$ of each hystogram in Figure 4.

In Figure 6, the evaluated SBH (dots)is reported as a function of the mean NC size. It increases with increasing average NC size, tending asymptotically to the ideal SBH value of a continuous $\mathrm{Au}$ film/SiC contact $(\sim 1.9 \mathrm{eV})$ [17]. This latter evidence indicates that the larger Au NCs (>7 nm) on $\mathrm{SiC}$ approach the behavior of the Schottky barriers formed by continuous Au films.

We based our interpretation of the SBH dependence on the NCs size considering the thermoionic transport theory through the MS barrier coupled with the concept of ballistic transport and the constant interaction (CI) model for the electron transport in few electrons quantum dots [18]. The process was schematized as indicated in the inset of Figure 6. For a forward (positively) biased tip, an additional electron from the substrate overcomes the $\mathrm{SBH}$ by thermoionic emission and falls on the lowest unoccupied energy level $\mu(N+1)$ within the 3D box containing $N$ electrons (NC). As the electron mean free path $\lambda_{e}$ for the considered electron kinetic energy in the Au NCs is ranging between [19] 10 and $20 \mathrm{~nm}$ (i.e., $\lambda_{e}$ larger than the average cluster dimension), the electron moves ballistically within the Au dot and it is collected to the tip in ohmic contact with the Au grain. Hence, the $\mathrm{Au} \mathrm{Nc/SiC} \mathrm{SBH}$ is given by $\Phi_{B}(N)=\Phi_{B 0}-$ $\Delta \mu(N)$, with $\Delta \mu(N)$ the energetic distance between $E_{F}$ and $\mu(N+1)$ (inset of Figure 6) and $\Phi_{B 0}$ the Schottky barrier height as defined for the macroscopic contact. According to the CI model of few electron quantum dots, $\Delta \mu(N)=$ $\left(e^{2} / C\right)+\Delta E$ where $E_{c}=e^{2} / C$ is the electrostatic energy ("charging energy") necessary to add or subtract one electron to the dot, taking into account the Coulomb interactions of that electron with all other electrons, in and outside the dot. We derived the capacitance $C$ as $C=C_{0}+n_{s} C_{c} ; C_{0}=2 \pi \varepsilon_{r} \varepsilon_{0} L$ is the self-capacitance of the dot, that is, the capacitance of a sphere of diameter $L$ embedded in a dielectric of constant $\varepsilon_{r}, C_{c} \approx\left(\pi^{3} \varepsilon_{r} \varepsilon_{0} L^{2}\right) / 4(s+L)$ is an approximated expression for the coupling capacitance between two nearest clusters, described as two spheres with the same diameter $L$ and sited at center-to-center distance $s+L(s$ is the surfacesurface distance between the two clusters). Finally, $n_{s}$ is the number of nearest neighbour clusters whose analytical expression is derived starting from the results exposed in Section 3.1. According to those results, the surface clusters density is expressed by $N_{s}(L)=3 Q \Omega / 4 \pi L^{3}$, where $Q$ is the $\mathrm{Au}$ atomic surface concentration. In a random distribution of the clusters on the surface, a circular symmetry around each fixed cluster can be assumed, so that $n_{s}=2 \pi(s+L) \sqrt{N_{s}}$. So:

$$
\begin{aligned}
\Phi_{B}(L)= & \Phi_{B 0}-\frac{e^{2}}{2 \pi \varepsilon_{r} \varepsilon_{0} L+(\sqrt{3 Q \Omega / 4 \pi})\left(\pi^{3} \varepsilon_{r} \varepsilon_{0} / 2\right) L^{1 / 2}} \\
& -\left(\frac{1}{3 \pi^{2}}\right)^{1 / 3}\left(\frac{\pi}{6 \Omega}\right)^{-1 / 3} \frac{\hbar^{2} \pi^{2}}{m^{*} L^{3}} .
\end{aligned}
$$

Clearly, $\Phi_{B}(L)$ tends to saturate to $\Phi_{B 0}$ for sufficiently large $L$ values, that is, the behavior of large NCs approaches that of the bulk material. By (3) it is evident that, decreasing $L$, the barrier height $\Phi_{B}$ becomes zero for a certain $L^{*}$ value, which in our case is $L^{*}=0.85 \mathrm{~nm}$. For $L<L^{*}, \Phi_{B}$ would be negative. Since $\Phi_{B}(L)=\Phi_{m}-\chi-\Delta \mu(L)=\Phi_{\text {meff }}(L)-$ $\chi$, a negative $\Phi_{B}$ value is equivalent to an effective metal work-function $\Phi_{\text {meff }}(L)$ lower than the electron affinity $\chi$ in 
the semiconductor. In such a situation, the Au NC/6H-SiC contact would be not any more a Schottky contact, but an ohmic one and the transport mechanism at the interface would change in consequence. Although the developed model is valid for $T=0$, it gives a good approximation even at room temperature (our experimental case) for which $\left(e^{2} / C\right) \gg k T$.

Comparing the theoretical prediction by (3) with the experimental data of Figure 6 using the values $\Phi_{B 0}=1.85 \mathrm{eV}$, $\varepsilon_{r}=1, Q=4.5 \times 10^{15} \mathrm{~cm}^{-2}$ (by RBS analyses), and $\Omega=$ $1.69 \times 10^{-29} \mathrm{~m}^{3}$, the continuous curve reported in Figure 6 is obtained. The only free fitting parameter was $m^{*}$. The best agreement was obtained for $m^{*}=0.08 \mathrm{~m}$. For NCs characterized by size larger than $10 \mathrm{~nm}$, it is evident that . As a consequence NCs with size larger than $10 \mathrm{~nm}$ acquire bulk properties. This is confirmed by other experimental evidences as $\mathrm{Au}$ NCs melting point and structural properties dependence on size [20].

\section{CONCLUSION}

The possibility of controlling and modeling size and size distribution of $\mathrm{Au}$ NCs deposited on $\mathrm{SiC}$ surface by process parameters such as thermal treatments has been demonstrated.

The clustering kinetic process and surface diffusion of $\mathrm{Au}$ on $\mathrm{SiC}$ substrates were experimentally characterized by Rutherford backscattering spectrometry, scanning electron microscopy, and atomic force microscopy. The evolution kinetics has been interpreted by classical models involving surface diffusion limited ripening of spherical threedimensional clusters on a substrate. From the mass transfer surface diffusion coefficients of gold on $\mathrm{SiC}$ hexagonal and $\mathrm{SiO}_{2}$ surfaces, determined in the $873 \mathrm{~K} \div 1073 \mathrm{~K}$ temperature range, activation energy of $(0.55 \pm 0.01) \mathrm{eV} /$ atom was obtained. The knowing of the details of the self-organization mechanisms of $\mathrm{Au} \mathrm{NCs}$ on $\mathrm{SiC}$ allowed us to fabricate nanoSchottky diodes with tunable electrical properties by tuning the parameters characterizing these mechanisms.

\section{REFERENCES}

[1] P. Moriarty, "Nanostructured materials," Report on Progress in Physics, vol. 64, no. 3, pp. 297-381, 2001.

[2] D. K. Ferry and S. M. Goodnick, Transport in Nanostructures, Cambridge University Press, London, UK, 1997.

[3] M. Henini, "Properties and applications of quantum dot heterostructures grown by molecular beam epitaxy," Nanoscale Research Letters, vol. 1, no. 1, pp. 32-45, 2006.

[4] H. E. Ruda, J. C. Polanyi, J. Yang, et al., "Developing 1D nanostructure arrays for future nanophotonics," Nanoscale Research Letters, vol. 1, no. 2, pp. 99-119, 2006.

[5] G. Schmid, Ed., Nanoparticles, Wiley-VCH, Weinheim, Germany, 2004.

[6] I. Beszeda, E. G. Gontier-Moya, and Á. W. Imre, "Surface Ostwald-ripening and evaporation of gold beaded films on sapphire," Applied Physics A, vol. 81, no. 4, pp. 673-677, 2005.

[7] A. Baldan, "Progress in Ostwald ripening theories and their applications to nickel-base superalloys-part I: Ostwald ripening theories," Journal of Materials Science, vol. 37, no. 11, pp. 2171-2202, 2002.

[8] I. M. Lifshitz and V. V. Slyozov, "The kinetics of precipitation from supersaturated solid solutions," Journal of Physics and Chemistry of Solids, vol. 19, no. 1-2, pp. 35-50, 1961.

[9] C. Wagner, "Theorie der Alterung von Niederschlägen durch Umlösen," Zeitschrift für Elektrochemie, vol. 65, pp. 581-591, 1961.

[10] M. Zinke-Allmang, L. C. Feldman, and M. H. Grabow, "Clustering on surfaces," Surface Science Reports, vol. 16, no. 8, pp. 377-463, 1992.

[11] Z. Wang and P. Wynblatt, "The equilibrium form of pure gold crystals," Surface Science, vol. 398, no. 1-2, pp. 259-266, 1998.

[12] Ph. Buffat and J.-P. Borel, "Size effect on the melting temperature of gold particles," Physical Review A, vol. 13, no. 6, pp. 2287-2298, 1976.

[13] B. K. Chakraverty, "Grain size distribution in thin films1. Conservative systems," Journal of Physics and Chemistry of Solids, vol. 28, no. 12, pp. 2401-2412, 1967.

[14] K. N. Tu, J. W. Mayer, and L. C. Feldman, Electronic Thin Film Science, Macmillan, New York, NY, USA, 1992.

[15] F. Giannazzo, F. Roccaforte, V. Raineri, and S. F. Liotta, "Transport localization in heterogeneous Schottky barriers of quantum-defined metal films," Europhysics Letters, vol. 74, no. 4, pp. 686-692, 2006.

[16] A. S. Groove, Physics and Technology of Semiconductor Devices, John Wiley \& Sons, New York, NY, USA, 1967.

[17] L. A. Kosyachenko, V. M. Sklyarchuk, and Ye. F. Sklyarchuk, "Electrical and photoelectric properties of Au-SiC schottky barrier diodes," Solid-State Electronics, vol. 42, no. 1, pp. 145151, 1998.

[18] L. P. Kouwenhoven, D. G. Austing, and S. Tarucha, "Fewelectron quantum dots," Reports on Progress in Physics, vol. 64, no. 6, pp. 701-736, 2001.

[19] A. Zangwill, Physics at Surfaces, Cambridge University Press, Cambridge, UK, 1988.

[20] M. B. Cortie and E. van der Lingen, "Catalytic gold nanoparticles," Materials Forum, vol. 26, pp. 1-14, 2002. 

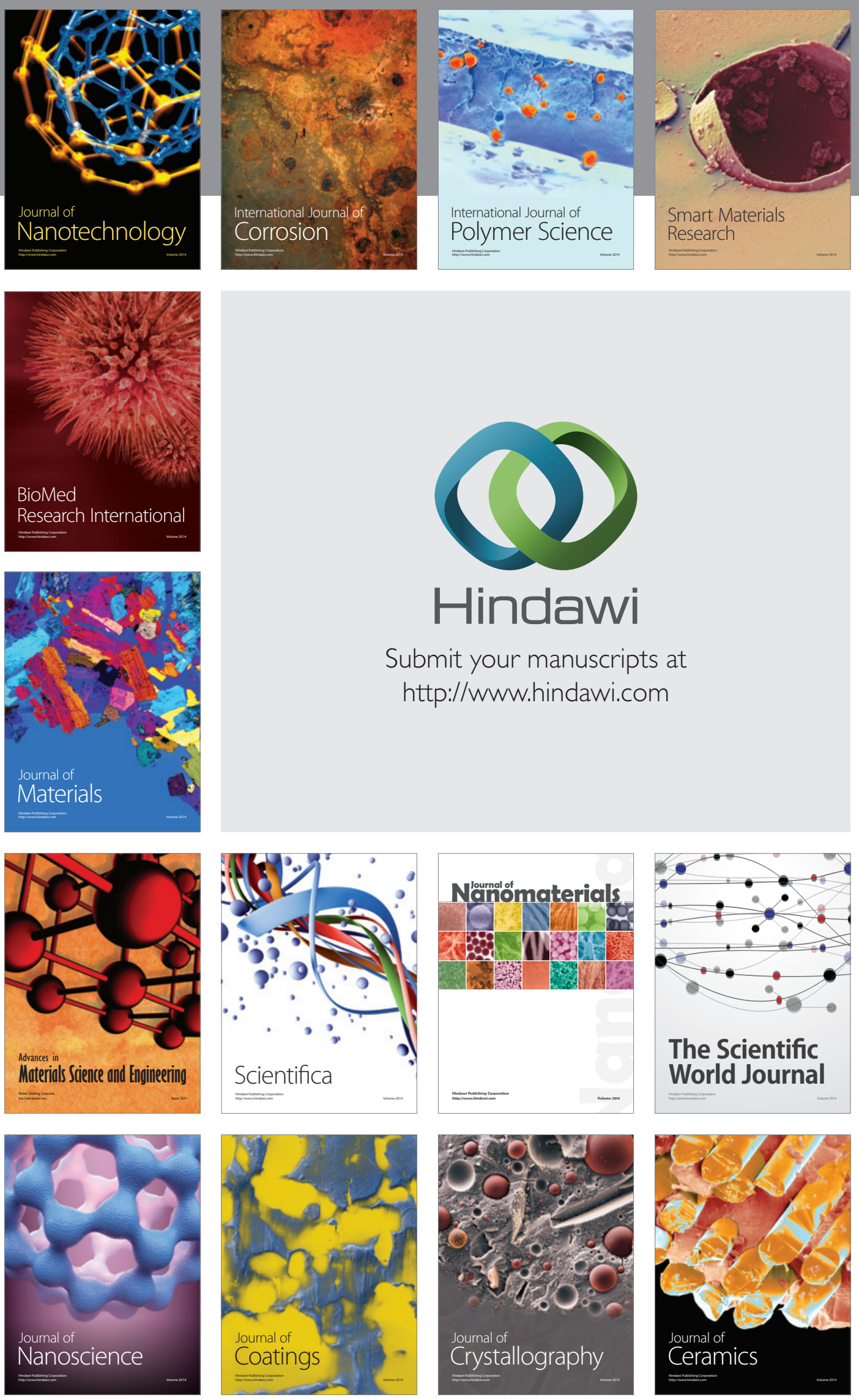

The Scientific World Journal

Submit your manuscripts at

http://www.hindawi.com

\section{World Journal}

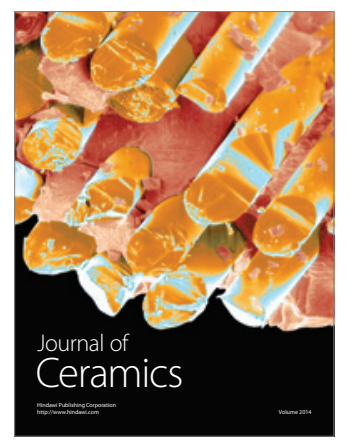

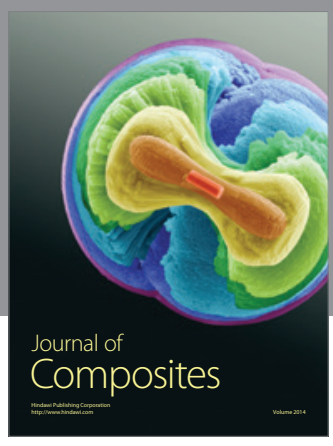
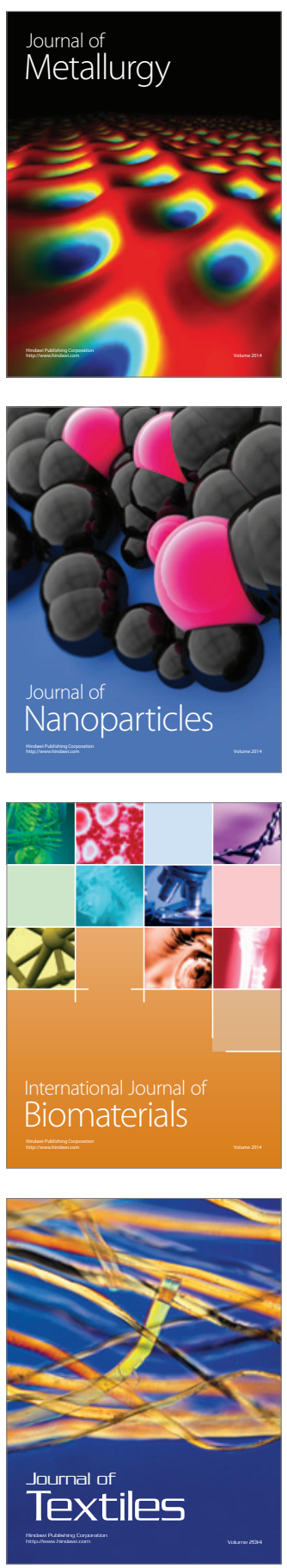